\title{
Fatores associados ao tabagismo em idosos residentes na cidade de Londrina, Brasil
}

\author{
Factors associated with the habit of smoking in elderly residents in Londrina city, Brazil
}

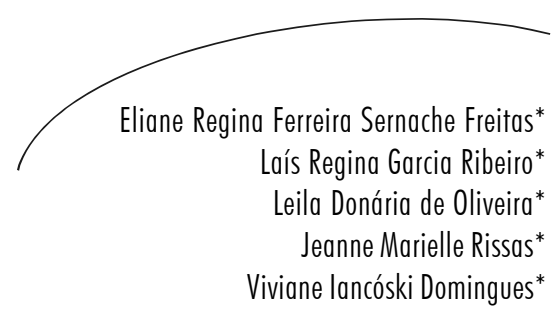

Objetivo: Descrever as características e fatores associados ao tabagismo em uma população idosa. Métodos: Estudo transversal realizado com 344 idosos ( $\geq 60$ anos) residentes na cidade de Londrina, estado do Paraná, em 2008. A variável dependente foi o tabagismo e as demais variáveis estudadas foram: fatores socioeconômicos, escolaridade, condição referida de saúde e uso de serviço de saúde. Os dados foram coletados por inquérito domiciliar. Estatísticas descritivas das variáveis do estudo foram utilizadas para avaliar associações entre o tabagismo (atual e passado) e as variáveis exploratórias. Resultados: A prevalência de tabagismo atual e passado foi de $23,8 \%$ e $37,6 \%$ entre os homens e de $12,7 \%$ e $21,8 \%$ entre as mulheres, respectivamente $(\mathrm{p}<0,0001)$. Entre os tabagistas atuais, não houve diferença entre homens e mulheres em relação ao consumo de cigarros diário e o grau de dependência do tabaco. A idade também não demonstrou diferença entre os grupos (fumante atual, ex-fumante e nunca fumante). Houve uma associação independente e negativa com escolaridade e condição socioeconômica (renda familiar) e associação positiva com percepção ruim ou muito ruim de saúde. Conclusão: $\mathrm{O}$ tabagismo constituiu um problema de saúde pública entre os idosos da comunidade estudada. A baixa escolaridade e a condição socioeconômica devem ser consideradas em programas específicos de cessação do tabagismo entre idosos.

\section{Abstract}

Objective: To describe the characteristics and associated factors of smoking among the elderly. Methods: Cross-sectional study carried out with 344 seniors $(\geq 60$ years old) living in the mid-sized, southern city of Londrina, Brazil in 2008. The dependent variable was smoking and the other studied variables were: socio-economic factors, education, referred health status and use the use of health services. Data were collected through household survey. Descriptive statistics of the variables of study and invariant data analysis were used to assess associations between the smoking habits of both current and former smokers and the exploratory variables. Results: The prevalence of current and past smoking was $23.8 \%$ and $37.6 \%$ among men, and
Palavras-chave:

Tabagismo. Idoso. Saúde do Idoso. Prevalência.

Escolaridade. Fatores

Socioeconômicos. Estudos

Transversais.

Universidade Norte do Paraná. Departamento de Fisioterapia, Londrina, PR, Brasil 
of $12.7 \%$ and $21.8 \%$ among women, respectively ( $p<0.0001)$. Among current smokers, there was no difference between men and women in relation to consumption of cigarettes per day and degree of dependence on tobacco. Age was not a determining factor among the groups (current smoker, former smoker and never having smoked) either. There was an independent and negative association with schooling and socioeconomic condition and positive association with poor health perception. Conclusion: Smoking was a public health concern among older adults in the studied community. Lower degrees of education and socioeconomic condition should be considered in the development of stop smoking programs among the elderly.
Key words: Smoking. Aged. Health of the Elderly. Prevalence. Educational Status. Socioeconomic Factors. Cross-Sectional Studies.

\section{INTRODUÇÃO}

O tabagismo, antes visto como um estilo de vida é atualmente reconhecido como uma dependência química que expõe os indivíduos a inúmeras substâncias tóxicas. Considerado como grande problema de saúde pública pela Organização Mundial de Saúde (OMS), o tabagismo deve ser encarado como uma pandemia, responsável por cerca de cinco milhões de mortes (quatro milhões de homens e um milhão de mulheres) por ano em todo o mundo. ${ }^{1}$

Não havendo uma mudança de curso da exposição mundial ao tabagismo, a OMS estima que o número de fumantes do ano 2000 a 2030 passará de 1,2 bilhão para 1,6 bilhão e que o número de mortes anuais atribuíveis ao tabagismo aumentará de 4,9 para 10 milhões, dos quais $70 \%$ ocorrerão nos países em desenvolvimento. ${ }^{2}$

Acredita-se que a prevalência de tabagismo entre idosos no Brasil seja em torno de $10 \%$ a $11 \%$ da população geral de fumantes. ${ }^{2}$ Porém, atualmente foi demonstrado que acima de 65 anos a prevalência de fumantes é de $11,5 \%$, mas que cima de 65 anos é de $8 \%$ da população de fumantes e $35,3 \%$ de ex-fumantes. ${ }^{3,4}$

O tabagismo entre idosos está associado ao maior risco de morbidade e mortalidade por doenças cardiovasculares, cerebrovasculares, respiratórias, diversos tipos de cânceres ${ }^{5,6}$ e por um custo social e financeiro alto para as instituições de saúde. ${ }^{2}$

O expressivo número de mortes que ocorrem a partir dos 65 anos, provenientes das doenças isquêmicas do coração e cerebrovasculares, tem o tabaco como o principal fator de risco. ${ }^{7} \mathrm{Na}$ Região Sul, a prevalência de tabagismo atual em idosos em 1998 foi de $15,8 \% .^{8}$

Os idosos que fumam apresentam algumas características que os diferenciam dos jovens, pois apresentam maior dependência da nicotina, geralmente fumam maior número de cigarros, fumam há mais tempo, o que pode aumentar sua dependência e dificultar cessação do hábito, e como consequência, têm mais problemas de saúde relacionados ao cigarro. ${ }^{9}$

Para se fazer qualquer planejamento estratégico visando-se à abordagem e controle do tabagismo em idosos, é necessário conhecer os motivos pelos quais os idosos fumam, a influência do ambiente familiar, socioeconômico e cultural sobre eles, os aspectos da dependência nicotínica e as interrelações das comorbidades dependentes do tabaco, como fatores determinantes na manutenção da qualidade de vida. ${ }^{10} \mathrm{~A}$ partir desses conhecimentos, deve-se procurar de maneira criteriosa uma melhor estratégia de tratamento ao idoso fumante, pois supõe-se que os idosos, que reconhecem no tabagismo os efeitos negativos sobre a sua saúde, tenham um maior desejo de cessação desse hábito. ${ }^{10}$

O objetivo do presente estudo foi descrever o tabagismo da população idosa residente na região central da cidade de Londrina-PR e os fatores associados ao tabagismo atual e passado, segundo as características socioeconômicas, escolaridade, saúde referida e uso dos serviços de saúde. 


\section{MÉTODOS}

Estudo transversal, envolvendo indivíduos com idade igual ou acima de 60 anos de ambos os sexos, não-institucionalizadas e residentes na região central da cidade de Londrina-PR. Os dados apresentados foram coletados de maio a agosto de 2008, mediante inquérito domiciliar que avaliou o tabagismo, as características socioeconômicas, escolaridade, condição referida de saúde e uso de serviço de saúde.

A cidade de Londrina está localizada na Região Sul do Brasil, no Estado do Paraná (PR). Possui uma área de unidade territorial de $1.651 \mathrm{~km}^{2}$, com 497.833 habitantes, de acordo com os dados do Instituto Brasileiro de Geografia e Estatística (IBGE) de 2007. ${ }^{11}$ A população de idosos com idade igual ou superior a 60 anos registrados em 39 Unidades Básicas de Saúde (UBS) do município (regiões Norte, Sul, Leste, Oeste e Centro) totaliza 44.710 idosos, sendo que a UBS Centro, tomada como população de referência para o cálculo da amostra, totaliza 8.706.

A amostra foi obtida de forma probabilística em dois estágios: no primeiro, foram sorteadas as ruas do centro da cidade de maneira sistemática; no segundo, sortearam-se os domicílios por numeração, considerando o início e o fim da rua e lados par e ímpar. Em cada uma das ruas, foram identificados pelo menos três idosos que preenchessem os critérios de inclusão do estudo: ter 60 anos ou mais, ter condições de responder ao instrumento de pesquisa, ser morador da área de abrangência da cidade e aceitar participar da pesquisa. Na ocorrência de identificação de um idoso que não preenchesse os critérios de inclusão, era repetido o mesmo processo de inquérito até encontrar-se um substituto que atendesse aos critérios definidos. Nos domicílios em que se encontraram dois ou mais idosos que respeitassem os critérios de inclusão, era realizado sorteio entre eles.

Determinou-se como aceitável entrevistar entre 200 e 400 idosos. Nessas circunstâncias, o erro de amostragem para estimar proporções do processo aleatório simples manteve-se abaixo de $5 \%$, indicando um número mínimo de 334 entrevistas para coeficiente de confiança igual a $95 \% .{ }^{12}$

O número mínimo, fixado entre 200 e 400 idosos, atendeu à necessidade de proteger o efeito de homogeneidade intrassetor censitário, ${ }^{13}$ tomado como unidade primária de amostragem da região central da cidade de Londrina-PR.

Foram contatados 547 idosos; 95 foram excluídos porque não tinham condições de responder às questões da entrevista e 108 não aceitaram participar do estudo (taxa de recusa de 19,7\%). Portanto, participaram da pesquisa 344 idosos. A variável dependente foi o tabagismo, sendo os participantes classificados em: nuncafumantes, ex-fumantes e fumantes atuais.

Segundo os critérios do OMS, ${ }^{14}$ definiu-se como fumante todo indivíduo que, por ocasião do inquérito, estivesse fumando qualquer tipo ou quantidade de tabaco, diariamente, há pelo menos seis meses; como ex-fumantes aqueles que, tendo sido fumantes, não tenham fumado qualquer tipo ou quantidade de tabaco nos últimos seis meses; e como não-fumantes, os que nunca tenham fumado, ou por pouco tempo ou de forma esporádica, qualquer tipo ou quantidade de tabaco, em qualquer período da vida, não sendo, portanto, considerados ex-fumantes.

O grau de dependência do tabaco foi avaliado pelo questionário de tolerância de Fagerström. ${ }^{15}$ O objetivo desse questionário é identificar e medir a dependência de nicotínica de acordo com a soma de pontos obtidos com as respostas referentes às seis perguntas (quadro 1), classificando a dependência da nicotínica em cinco graus: muito baixa ( 0 a 2), baixa (3 a 4), média (5), elevada (6 a 7) e muito elevada ( 8 a 10). ${ }^{16}$

Para o ponto de corte entre fumantes com baixa e elevada dependência do tabaco, foi adotada a mediana, cujo valor foi seis. Assim os fumantes que somaram acima de seis pontos (dependência elevada ou muito elevada) foram considerados como: elevada dependência de nicotina (EDN), e aqueles que somaram menos de seis pontos (dependência média, baixa ou muito baixa) foram considerados como: baixa dependência de nicotina (BDN). 
As demais variáveis foram características sociodemográficas, como idade, sexo, situação conjugal, anos completos de escolaridade, renda familiar, condição autorreferida de saúde (percepção de saúde boa, regular, ruim, muito ruim); e uso dos serviços de saúde (consultas médicas e internações nos últimos 12 meses).

Foram utilizadas estatísticas descritivas das variáveis do estudo pelo cálculo das médias, medianas e desvios-padrão. A análise dos dados foi realizada utilizando-se o teste Qui-quadrado de Pearson e Qui-quadrado para tendência linear (para proporções), avaliando-se as associações entre o tabagismo (atual e passado) e as variáveis exploratórias. Estabeleceu-se para nível de significância estatística o valor de $5 \%(\mathrm{p}<0,05)$ e intervalo de confiança de $95 \%$. Para análise dos dados, foram utilizados os programas Statistical Package of Social Science (SPSS) na versão 14.0 e BioEstat na versão 4.0

O trabalho foi aprovado pelo Comitê de Ética e Pesquisa da Universidade Norte do Paraná - CEP/ UNOPAR (protocolo $n^{\circ}$ 0394/07), e assinado o termo de consentimento livre e esclarecido pelos envolvidos no estudo, resguardando-se a privacidade e confidencialidade dos idosos.

Quadro 1 - Perguntas do Questionário de Tolerância de Fagerström. Londrina, PR, 2008.

\begin{tabular}{|c|c|c|}
\hline \multirow[t]{5}{*}{1} & Pergunta $\mathrm{n}^{\mathbf{0}}$ 1: Quanto tempo após acordar você fuma o seu primeiro cigarro? & \\
\hline & - Dentro de 5 minutos & 3 \\
\hline & - Entre 6 e 30 minutos & 2 \\
\hline & - Entre 31 e 60 minutos & 1 \\
\hline & - Após 60 minutos & 0 \\
\hline \multirow[t]{3}{*}{2} & $\begin{array}{l}\text { Pergunta } n^{\circ} \text { 2: Você acha difícil não fumar em locais onde o fumo é proibido (como igrejas, } \\
\text { biblioteca, etc)? }\end{array}$ & \\
\hline & - $\operatorname{Sim}$ & 1 \\
\hline & - Não & 0 \\
\hline \multirow[t]{3}{*}{3} & $\begin{array}{l}\text { Pergunta } n^{\circ} \text { 3: Qual o cigarro do dia traz mais satisfação (ou que mais detestaria deixar de } \\
\text { fumar)? }\end{array}$ & \\
\hline & - O primeiro da manhã & 1 \\
\hline & - Outros & 0 \\
\hline \multirow[t]{5}{*}{4} & Pergunta $n^{\circ} 4:$ Quantos cigarros você fuma por dia? & \\
\hline & - 10 ou menos & 0 \\
\hline & - $\quad 11$ a 20 & 1 \\
\hline & - 21 a 31 & 2 \\
\hline & - $\quad 31$ ou mais & 3 \\
\hline \multirow[t]{3}{*}{5} & $\begin{array}{l}\text { Pergunta } n^{\circ} \text { 5: Você fuma mais frequentemente pela manhã (nas primeiras horas do dia) que } \\
\text { no resto do dia? }\end{array}$ & \\
\hline & - $\quad \operatorname{Sim}$ & 1 \\
\hline & - Não & 0 \\
\hline \multirow[t]{3}{*}{6} & $\begin{array}{l}\text { Pergunta } \mathbf{n}^{\mathbf{0}} \text { 6: Você fuma mesmo quando está tão doente que precisa ficar de cama a maior } \\
\text { parte do tempo? }\end{array}$ & \\
\hline & - $\quad \operatorname{Sim}$ & 1 \\
\hline & - Não & 0 \\
\hline
\end{tabular}




\section{RESULTADOS}

Entre os 344 idosos participantes da pesquisa, $202(58,7 \%)$ eram homens e $142(41,3 \%)$ mulheres. A média de idade foi de $69,7( \pm 7,5)$ anos, variando entre 60 a 93 anos.

A tabela 1 mostra algumas características do tabagismo entre os idosos de acordo com o gênero. A prevalência de tabagismo atual e passado foi de $23,8 \%$ e $37,6 \%$ entre os homens, e $12,7 \%$ e $21,8 \%$ entre as mulheres $(\mathrm{p}<0,0001)$. Entre os fumantes atuais, $24,3 \%$ dos homens e $34,5 \%$ das mulheres consumiam menos de 10 cigarros por dia; porém, entre os homens, o maior percentual foi de $43,2 \%$, os quais consumiam de 11 a 20 cigarros por dia $(p=0,902)$.

Elevada dependência de nicotina (EDN) foi encontrada em 43,2\% dos homens e em $27,6 \%$ das mulheres, mas esses resultados não apresentaram diferença estatística significante $(\mathrm{p}=0,1894)$ (tabela 1).

Tabela 1 - Características do tabagismo entre idosos de acordo com o gênero. Londrina, PR, 2008.

\begin{tabular}{lccc}
\hline Variável & $\begin{array}{c}\text { Homem } \\
\mathrm{N}=202\end{array}$ & $\begin{array}{c}\text { Mulher } \\
\mathrm{N}=142\end{array}$ & $\begin{array}{c}\text { Total } \\
\mathrm{N}=344\end{array}$ \\
\hline Tabagismo (\%) & & & \\
Nunca fumante & $78(38,6)$ & $93(65,5)$ & $171(49,7)$ \\
Ex-fumante & $76(37,6)$ & $31(21,8)$ & $107(31,1)$ \\
Fumante atual & $48(23,8)$ & $18(12,7)$ & $66(19,2)$ \\
Número de cigarros fumados por & & $\mathrm{p}<0,0001^{*}$ & \\
dia entre os fumantes atuais (\%) & & & \\
$\leqslant 10$ & $9(24,3)$ & $10(34,5)$ & $19(28,8)$ \\
$11-20$ & $16(43,2)$ & $7(24,1)$ & $23(34,8)$ \\
$21-30$ & $6(16,2)$ & $6(20,7)$ & $12(18,2)$ \\
$\geqslant 30$ & $6(16,2)$ & $6(20,7)$ & $12(18,2)$ \\
& & $\mathrm{p}=0,902 * *$ & \\
Grau de dependência do tabaco & $16(43,2)$ & $8(27,6)$ & $29(43,9)$ \\
EDN & $21(56,8)$ & $21(72,4)$ & $37(56,1)$ \\
BDN & & $\mathrm{p}=0,1894 *$ & \\
& & & \\
\hline
\end{tabular}

EDN: Elevada dependência de nicotina; BDN: Baixa dependência de nicotina

*Teste Qui-quadrado de Pearson

** Teste Qui-quadrado para tendência linear

A tabela 2 apresenta a distribuição do tabagismo de acordo com as características sociodemográficas. Na faixa etária de 60 a 64 anos, a prevalência de fumantes foi de $39,4 \%$ e de 26,2\% para ex-fumantes. Apesar de o estudo não ter apresentado diferença significativa entre os grupos de nunca fumantes, ex-fumantes e fumantes atuais em relação às faixas etárias $(p=0,365)$, houve decréscimo de fumantes atuais e um aumento de ex-fumantes com o aumento da idade.
Não foi observada nenhuma associação entre o tabagismo e a situação conjugal $(\mathrm{p}=0,365)$, mas uma associação negativa foi observada em relação à renda familiar $(p=0,009)$ e escolaridade $(p=0,015)$.

A distribuição do tabagismo e os indicadores da condição de saúde são apresentados na tabela 3. Significante associação foi encontrada entre o tabagismo e a piora da percepção autorreferida de saúde. Dos tabagistas atuais, $16,7 \%$ referiram 
ter saúde ruim e $3,0 \%$, muito ruim $(p<0,0001)$; $71,2 \%$ procuraram o serviço de saúde para consulta médica $(p<0,0001)$ e $69,7 \%$ foram hospitalizados $(\mathrm{p}<0,0001)$ pelo menos uma vez no último ano. Dos ex-tabagistas, $8,4 \%$ referiram ter saúde ruim, $73,8 \%$ consultaram o médico e $69,2 \%$ foram hospitalizados no último ano $(\mathrm{p}<0,0001)$.

Tabela 2. Distribuição dos idosos de acordo com as características sóciodemográficas em relação ao tabagismo. Londrina, PR, 2008.

\begin{tabular}{lcccc}
\hline Variáveis & $\begin{array}{c}\text { Nunca fumante } \\
(\mathrm{N}=171) \\
\%\end{array}$ & $\begin{array}{c}\text { Ex-fumante } \\
(\mathrm{N}=107) \\
\%\end{array}$ & $\begin{array}{c}\text { Fumante atual } \\
(\mathrm{N}=66) \\
\%\end{array}$ & $\begin{array}{c}\text { Total } \\
(\mathrm{N}=344) \\
\%\end{array}$ \\
\hline Faixa etária (anos) & 29,2 & 26,2 & 39,4 & 30,2 \\
$60-64$ & 24,0 & 22,4 & 25,8 & 23,8 \\
$65-69$ & 19,9 & 19,6 & 12,1 & 18,3 \\
$70-74$ & 15,2 & 15,0 & 12,1 & 14,6 \\
$75-79$ & 11,7 & 16,8 & 10,6 & 13,1 \\
$\geqslant 80$ & & $\mathrm{P}=0,365^{* * *}$ & & \\
Situação conjugal & & & & \\
Casado & 66,7 & 57,0 & 59,1 & 62,2 \\
Outro & 33,3 & 43,0 & 40,9 & 37,8 \\
& & $\mathrm{p}=0,229 *$ & & \\
Renda Familiar (Salários) & & & & 21,2 \\
0 & 15,8 & 26,2 & 27,3 & 44,8 \\
$1-2$ & 45,0 & 43,0 & 47,0 & 22,4 \\
$3-4$ & 25,7 & 18,7 & 19,7 & \\
$\geqslant 5$ & 13,5 & 12,1 & 6,0 & \\
& & $\mathrm{p}=0,009^{* * *}$ & & 31,8 \\
Escolaridade (anos) & & & & 34,8 \\
Nunca estudou & 15,2 & 20,6 & 31,8 & 13,2 \\
$1-4$ & 47,4 & 43,0 & 34,8 & 1,6 \\
$5-8$ & 15,2 & 8,4 & 18,2 & \\
$9-11$ & 17,5 & 20,5 & 13,6 & \\
$\geqslant 12$ & 4,7 & 7,5 & 1,6 & \\
& & $\mathrm{p}=0,015^{* * *}$ & & \\
\hline
\end{tabular}

*Teste do Qui-quadrado de Pearson

** Teste do Qui-quadrado para tendência linear 
Tabela 3 - Indicadores das condições de saúde dos idosos segundo o tabagismo. Londrina, PR, 2008.

\begin{tabular}{|c|c|c|c|c|}
\hline Variáveis & $\begin{array}{c}\text { Nunca fumante } \\
(\mathrm{N}=171) \\
\%\end{array}$ & $\begin{array}{c}\text { Ex-fumante } \\
(\mathrm{N}=107) \\
\%\end{array}$ & $\begin{array}{c}\text { Fumante atual } \\
(\mathrm{N}=66) \\
\%\end{array}$ & $\begin{array}{c}\text { Total } \\
(\mathrm{N}=344) \\
\%\end{array}$ \\
\hline \multicolumn{5}{|c|}{ Percepção auto referida de saúde } \\
\hline Boa & 43,8 & 21,5 & 45,5 & 37,2 \\
\hline Regular & 51,5 & 70,1 & 34,8 & 54,1 \\
\hline Ruim & 4,7 & 8,4 & 16,7 & 8,1 \\
\hline Muito ruim & 0,0 & $\begin{array}{c}0,0 \\
p<0,0001 *\end{array}$ & 3,0 & 0,6 \\
\hline \multicolumn{5}{|c|}{$\begin{array}{l}\text { Consultou o médico pelo menos } \\
\text { uma vez no ultimo ano }\end{array}$} \\
\hline Sim & 33,9 & 73,8 & 71,2 & 69,5 \\
\hline Não & 66,1 & $\begin{array}{c}26,2 \\
p<0,0001 * *\end{array}$ & 28,8 & 30,5 \\
\hline \multicolumn{5}{|c|}{$\begin{array}{l}\text { Foi hospitalizado pelo menos uma } \\
\text { vez no ultimo ano }\end{array}$} \\
\hline Sim & 24,0 & 69,2 & 69,7 & 46,8 \\
\hline Não & 76,0 & $\begin{array}{c}30,8 \\
p<0,0001 \% *\end{array}$ & 30,3 & 53,2 \\
\hline
\end{tabular}

*Teste do Qui-quadrado de Pearson

**Teste do Qui-Quadrado

\section{DISCUSSÃO}

O resultado deste estudo mostrou grande diferença no tabagismo entre homens e mulheres. A prevalência do tabagismo atual entre os homens foi o dobro da registrada entre as mulheres, resultado este semelhante a outros estudos. ${ }^{3,17-21}$ Nos países em desenvolvimento, os fumantes constituem $48 \%$ da população masculina e apenas $7 \%$ da feminina, apresentando predomínio masculino também no índice de mortalidade atribuível ao tabaco. ${ }^{18} \mathrm{~A}$ frequência do tabagismo foi de $16,2 \%$ no conjunto da população adulta das 27 cidades brasileiras estudadas pelo INCA, sendo também mais disseminado o tabagismo entre homens do que entre as mulheres, com exceção de Goiânia e Rio de Janeiro, onde a frequência de fumantes foi semelhante nos dois sexos. ${ }^{3}$ No entanto, a menor prevalência do tabagismo observada entre mulheres idosas deve ser examinada com prudência, devido à tendência do aumento desse hábito entre as mulheres mais jovens. Esse fato foi registrado nas cidades brasileiras economicamente mais desenvolvidas (regiões Sul e Sudeste), nas quais essa prevalência já se aproxima daquela observada na população masculina, ${ }^{20}$ o que aponta para uma ampliação deste índice em mulheres idosas nas próximas décadas. $^{10}$

Apesar de não ter apresentado significância estatística, o maior número de cigarros consumidos por dia entre os homens foi de 11 a $20(43,2 \%)$, enquanto que entre as mulheres foi abaixo de 10 cigarros/dia (34,5\%). De acordo com o Instituto Nacional de Câncer, ${ }^{20}$ em estudo realizado em 15 capitais e Distrito Federal (20022003), a análise da distribuição de fumantes segundo o número de cigarros fumados ao dia mostrou que a maior quantidade de cigarros consumidos encontrava-se na faixa de 1 a 10, seguida da de 11 a $20 .{ }^{22} \mathrm{O}$ consumo maior de cigarros entre os homens do presente estudo é semelhante ao observado na população norteamericana. ${ }^{23}$ No entanto, o consumo diário de cigarros na população idosa da cidade de Londrina foi menor do que a encontrada nos Estados Unidos $^{23}$ e Canadá. ${ }^{24}$ Nas três populações estudadas no Canadá (1985, 1986 e 1989), a 
proporção do número de cigarros consumidos em idosos ( $\geq 65$ anos) que fumavam mais de 20 cigarros por dia variou de $52 \%$ a $61 \%$ entre os homens e de $30 \%$ a $38 \%$ entre as mulheres. ${ }^{24}$ No entanto, o resultado de estudo realizado em $2007^{21}$ observou que o número de cigarros consumidos por homens brasileiros com $\leq 64$ anos se reduziu significantemente de 1983 a 2003, mas tal redução foi mínima entre as mulheres. O mesmo estudo observou que em indivíduos com idade $\geq$ de 65 anos, o número de cigarros consumidos ao dia permaneceu constante entre os homens e aumentou entre as mulheres.

A distribuição do tabagismo, segundo a faixa etária no presente estudo, demonstrou um decréscimo da frequência de fumantes com o aumento da idade. O oposto foi demonstrado no grupo de ex-fumantes, onde houve aumento linear da frequência de ex-fumantes com o aumento da idade. Alguns estudos ${ }^{3,22}$ demonstraram que, em ambos os sexos, a frequência de fumantes tende a aumentar com a idade até os 54 anos, seguindose um decréscimo em indivíduos idosos com 65 anos ou mais. Para o conjunto da população estudada em 27 cidades brasileiras, a frequência de ex-fumantes aumentou sistematicamente com a idade e foi semelhante entre homens e mulheres. ${ }^{3}$ Entretanto, a redução da prevalência de tabagismo com a idade não simboliza uma redução da média absoluta de idosos fumantes, pois, com o aumentando da prevalência de idosos no Brasil e no mundo, a tendência é caminhar para um aumento de idosos fumantes, como foi observado nos Estados Unidos no período de1965 a $1994 .{ }^{25}$

Estar na dependência nicotínica significa usar compulsivamente o fumo, ser incapaz de parar de fumar ou de manter-se sem fumar depois de parar, ou ter dificuldade de tentar parar por aparição de síndrome de abstinência e/ou desejo imperioso de fumar. ${ }^{26}$ Tanto a dependência nicotínica como sua síndrome de abstinência estão classificadas como doenças com critérios bem definidos. ${ }^{26}$ No presente estudo, o grau de dependência do tabaco, avaliado pela aplicação do questionário de tolerância de Fagerström, não apresentou diferença significativa entre homens e mulheres, mas a proporção de homens com EDN
$(43,2 \%)$ foi muito maior em comparação com as mulheres (27,6\%). Estudo realizado em quatro capitais brasileiras (Rio de Janeiro, São Paulo, Porto Alegre e Recife), com indivíduos entre $18 \mathrm{e}$ 64 anos, encontrou como resultado, em relação ao grau de dependência de nicotina, uma classificação de baixa a moderada dependência. ${ }^{27}$

A situação conjugal não demonstrou ser um fator que poderia influenciar o tabagismo nos resultados encontrados. Contudo, persistem algumas controvérsias em relação à influência da situação conjugal e o tabagismo em adultos mais velhos. $\mathrm{Na}$ Califórnia, foi demonstrado que o tabagismo é mais frequente entre homens e mulheres não casados no grupo de idade entre 50 e 65 anos. ${ }^{28}$ Também existem controvérsias em relação à situação conjugal e ao ato de deixar de fumar, pois em duas das quatro localidades dos Estados Unidos em que foi conduzido um estudo, foi demonstrado que o ato de deixar de fumar era mais frequente entre os adultos mais velhos que já haviam sido casados, enquanto que nas duas outras localidades o oposto foi encontrado..$^{23}$ Algumas hipóteses têm sido consideradas para explicar a associação entre tabagismo e a situação conjugal, tais como o maior apoio social observado entre casais, fato que favorece a cessação do tabagismo. ${ }^{28}$ A teoria está apoiada no fato de que os indivíduos casados têm uma maior possibilidade de receber aconselhamento e tratamento médico e apoio para cessação do tabagismo quando comparados com indivíduos que vivem sozinhos. ${ }^{29}$

Foi demonstrado no presente estudo uma forte relação do tabagismo com a baixa condição socioeconômica. Essa associação pode ser esclarecida pelo grande número de indivíduos com baixa condição socioeconômica, que começam a fumar muito jovens e, desta forma, explicar o alto grau de dependência nessa população, assim como a grande dificuldade para deixar o hábito, devido à baixa motivação e à escassez de recursos. ${ }^{21,30}$

A baixa escolaridade (menos de quatro anos de estudo) correlacionou-se com o maior número de fumantes (atuais e ex-fumantes), e os que apresentaram menor hábito fumante foram aqueles com maior nível de instrução 
( $\geq 12$ anos de estudo). Dados da literatura mostram que quanto menor o nível de escolaridade, maior o tabagismo. ${ }^{3,20,21,30,31}$ Nos Estados Unidos, a associação de tabagismo com escolaridade foi mais fraca entre idosos comparados com indivíduos jovens (18-64 anos), mostrando diferenças extremas de escolaridade ( $<9$ e $>15$ anos de estudo) nessa população. ${ }^{32}$ No Brasil, segundo informações do INCA,${ }^{20}$ foi possível observar que o tabagismo também é maior entre os grupos de menor escolaridade (ensino fundamental incompleto) que entre os de maior escolaridade (ensino fundamental completo e mais). ${ }^{20} \mathrm{~A}$ frequência de fumantes diminui uniforme e intensamente com o aumento da escolaridade em ambos os sexos, alcançando $24,2 \%$ e $14,7 \%$ dos homens e das mulheres com até oito anos de escolaridade e $14,4 \%$ e $9,1 \%$ dos homens e das mulheres com 12 ou mais anos de escolaridade, respectivamente. ${ }^{3}$

Em relação à autoavaliação de saúde, o resultado do estudo demonstrou uma associação positiva do tabagismo atual e passado com a percepção de pouca saúde. Houve também forte correlação entre o

\section{REFERÊNCIAS}

1. World Health Organization. World Health Report 2002. Reducing risks, promoting healthy life. Geneva: WHO, 2002.

2. World Health Organization. Building blocks for tobacco control: a handbook. Geneva: WHO; 2004.

3. Brasil. Vigitel Brasil 2006. Vigilância de Fatores de Risco e Proteção para Doenças Crônicas por Inquérito Telefônico. Brasilia: Ministério da Saúde; 2007. 92p. [Acesso em 20/03/2009]. Disponível em: URL: http://

bvsms.saude.gov.br/bvs/publicacoes/ relatorio_vigitel_2006_marco_2007.pdf.

4. Moura EC, Moraes Neto OL, Malta DC, Moura L, Silva NN, Bernal R, Claro RM, Monteiro CA. Vigilância de fatores de risco para doenças crônicas por inquérito telefônico nas capitais dos 26 estados brasileiros e no Distrito Federal (2006). Rev Bras Epidemiol 2008 may;11(1): 20-37. tabagismo atual e passado e a procura dos serviços de saúde em relação às consultas médicas e hospitalizações nos últimos 12 meses. A autopercepção de saúde é um consistente indicador de mortalidade na população idosa. ${ }^{33}$ Além do aumento do risco de morte, ${ }^{9,33}$ alguns estudos têm demonstrado que o tabagismo também reduz a qualidade de vida de idosos, principalmente quando associado à percepção de pouca saúde. , $23,37,32,34,35^{2}$

\section{CONCLUSÃO}

Na comunidade estudada, o tabagismo mostrou ser uma preocupação de saúde pública, pois o tabagismo teve grande impacto na percepção das condições de saúde e no uso dos serviços de saúde entre os indivíduos estudados. Sendo assim, nos programas de cessação do tabagismo entre idosos devem ser ressaltadas a baixa escolaridade e condição socioeconômica, pois isso adverte para as dificuldades que podem ser encontradas em um programa de redução da prevalência do tabagismo nessa população. Dessa forma, programas específicos para redução e/ou cessação do tabagismo para população idosa deveriam considerar esses resultados.

5. Ostbye T, Taylao DH, Jung SH. A longitudinal study of the effects of tobacco smoking and other mofifiable risk factors of ill health in middle-aged and old Americans: results from the Health and Retirement Study and Asset and Health Dynamics among the Oldest Old Survey. Prev Med 2002; 34:334-45.

6. Tessier JF, Nejjari C, Letenneur L, Barberger Gateau P, Dartigues JF, Salamon R. Smoking and eight-year mortality in an elderly cohort. Int J Tuberc Lung Dis 2000; 4:698-704.

7. González Enríquez J, Villar Alvarez F, Banegas Banegas JR, Rodríguez Artalejo F, Martín Moreno JM. Tendencia de la mortalidad atribuible al tabaquismo en España, 1978-1992: 600.000 muertes en 15 años. Med Clin (Barc). 1997;109:577-82.

8. Horta BL, Ramos Ede O, Victora CG. Determinants of the smoking habit in the city of Pelotas, Brazil. Bol Oficina Sanit Panam 1992 Aug;113(2):131-6. 
9. Cox JL. Smoking cessation in the elderly patient. Clin Chest Med 1993; 14: 423-8.

10. Peixoto SV, Firmo JOA, Lima-Costa MF. Condições de saúde e tabagismo entre idosos residentes em duas comunidades brasileiras (Projetos Bambuí e Belo Horizonte). Cad. Saúde Pública 2006; 22(9): 1925-34.

11. Ministério do Planejamento, Orçamento e Gestão. Instituto Brasileiro de Geografia e Estatística. Contagem da população 2007. Área de unidade territorial: Brasil, regiões geográficas e unidades federativas. Disponível em URL: http://www.ibge.gov.br.

12. Silva NN. Amostragem probabilística: Um curso introdutório. $2^{\mathrm{a}}$ ed. rev. São Paulo: EDUSP; 2000.

13. Kish L. Survey Sampling. New York: John Wiley \& Sons; 1969.

14. World Health Organization. Guidelines for the conduct of the tobacco smoking surveys of the general population: report of a meeting. Geneva, 1992. (N/WHO/SMO/83.4).

15. Heatherton TF, Kozlowski LT, Frecker RC, Fagerström KO. The Fagerström Test for Nicotine Dependence: a revision of the Fagerström Tolerance Questionnaire. Br J Addict 1991; 86(9): 1119-27.

16. Halty AS, Huttner MD, Isabel COM, Santos V, Martins G. Análise da utilização do questionário de tolerância de Fagerström (QTF) como instrumento de medidor de dependência de nicotina. J Bras Pneumol 2002;28(4): 180-86.

17. Oliveira AF, Valente JG, Leite IC. Aspectos da mortalidade atribuível ao tabaco: revisão sistemática. Rev. Saúde Pública 2008;2(2): 335-45.

18. Saueressig S, Hekman P, Consoni P, Menezes HS. Prevalência de tabagismo em idosos atendidos pelo Programa de Saúde da Família em Camaquã - RS. Rev. AMRIGS 2007;51(3): 173-79.

19. Correa PCRP, Barreto SM, Passos VMA. Methods for estimating smoking attributable Mortality: a review. Epidemiol Serv Saúde 2008;17(1): 43-57.

20. INCA/SVS. Instituto Nacional de Câncer/ Secretaria de Vigilância à Saúde. Inquérito Domiciliar Sobre Comportamentos de Risco e Morbidade Referida de Doenças e Agravos Nãotransmissíveis.[Acesso em 2009 mar 20] disponível em: URL: http://www.inca.gov.br/ vigilancia/fatores_de_risco.html.

21. Monteiro CA, Cavalcante TM, Moura EC, Claro RM, Szwarcwald CL. Population-based evidence of strong decline in the prevalence of smokers in Brasil (1989-2003). Bulletin of the World Health Organization 2007 july; 85(7):527-34.

22. Ministério da Saúde/Instituto Nacional de Câncer/Secretaria de Vigilância em Saúde. Inquérito domiciliar sobre comportamentos de risco e morbidade referida de doenças e agravos não transmissíveis: Brasil, 15 capitais e Distrito Federal, 2002-2003. Rio de Janeiro: Instituto Nacional de Câncer; 2004.

23. Colsher PL, Wallace RB, Pomrehn PR, LaCroix AZ, Cornoni-Huntley J, Blazer D, Scherr PA, Berkman L, Hennekens CH. Demographic and health characteristics of elderly smokers: results from established populations for epidemiologic studies of the elderly. Am J Prev Med. 1990 MarApr,6(2):61-70.

24. Maxwell CJ, Hirdes JP. The prevalence of smoking and implications for quality of life among the community-based elderly. Am J Prev Med. 1993 Nov-Dec,9(6):338-45.

25. Husten CG, Shelton DM, Chrismon JH, Lin YC, Mowery P, Powell FA. Cigarette smoking and smoking cessation among older adults: United States, 1965-94. Tob Control Autumn 1997 ; 6(3):175-80.

26. American Psychiatric Association. Diagnostic and statistical manual of mental disorders. $4^{\text {th }}$ ed. Washington, 1994.

27. Gigliotti A, Laranjeira R. Hábitos, atitudes e crenças de fumantes em quatro capitais brasileiras. Rev. Bras. Psiquiatr 2005;27(1): 37-44.

28. King AC, Taylor CB, Haskell WL. Smoking in older women. Is being female a 'risk factor' for continued cigarette use? Arch Intern Med. 1990 Sep;150(9):1841-6.

29. Ossip-Klein DJ, McIntosh S, Utman C, Burton K, Spada J, Guido J. Smokers ages 50+: who gets physician advice to quit? Prev Med. 2000 Oct;31(4):364-9.

30. Peixoto SV, Firmo JO, Lima-Costa MF. Factors associated to smoking habit among older adults (The Bambuí Health and Aging Study). Rev Saúde Pública. 2005 Oct;39(5):746-53. Epub 2005 Oct 24.

31. Little D. Review of Smoking in the Elderly. Geriatrics \& Aging 2002;9(6): 9-12.

32. Maxwell CJ, Dalby DM, Slater M, Patten SB, Hogan DB, Eliasziw M, Hirdes JP. The prevalence and management of current daily pain among older home care clients. Pain 2008 Aug 15;138(1):208-16. Epub 2008 May 29. 
33. Idler EL, Benyamini Y. Self-rated health and mortality: a review of twenty-seven community studies. J Health Soc Behav. 1997 Mar;38(1):2137. Review.

34. Husten CG, Shelton DM, Chrismon JH, Lin YC, Mowery P, Powell FA. Cigarette smoking and smoking cessation among older adults: United States, 1965-94. Tob Control. 1997 Autumn;6(3):175-80.

35. Hirdes JP, Maxwell CJ. Smoking cessation and quality of life outcomes among older adults in the Campbell's Survey on Well-Being. Can J Public Health. 1994 Mar-Apr;85(2):99-102.

Recebido: 03/9/2009

Revisado: 01/3/2010

Aprovado: 08/3/2010 
